\title{
Clinical Phenotype of a First Unprovoked Acute Pulmonary Embolism Associated with Antiphospholipid Antibody Syndrome
}

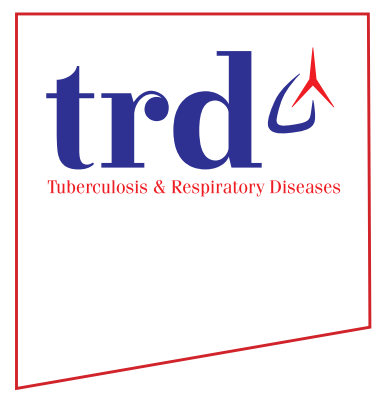

\author{
Yong Sub Na, M.S. ${ }^{1}{ }^{(\mathbb{D}}$, Seongsoo Jang, M.D. ${ }^{2}$, Seokchan Hong, M.D. ${ }^{3}$, Yeon Mok Oh, M.D. ${ }^{4}$, \\ Sang Do Lee, M.D. ${ }^{4}$ and Jae Seung Lee, M.D. ${ }^{5}$ (i) \\ ${ }^{1}$ Department of Pulmonary and Critical Care Medicine, Chosun University Hospital, Gwangju, Departments of ${ }^{2}$ Laboratory \\ Medicine, ${ }^{3}$ Rheumatology, and ${ }^{4}$ Pulmonary and Critical Care Medicine, Asan Medical Center, University of Ulsan College of \\ Medicine, Seoul, ${ }^{5}$ Department of Pulmonary and Critical Care Medicine, Center for Pulmonary Hypertension and Venous \\ Thrombosis, Asan Medical Center, University of Ulsan College of Medicine, Seoul, Korea
}

Background: Antiphospholipid antibody syndrome (APS), an important cause of acquired thrombophilia, is diagnosed when vascular thrombosis or pregnancy morbidity occurs with persistently positive antiphospholipid antibodies (aPL). APS is a risk factor for unprovoked recurrence of pulmonary embolism (PE). Performing laboratory testing for aPL after a first unprovoked acute PE is controversial. We investigated if a specific phenotype existed in patients with unprovoked with acute PE, suggesting the need to evaluate them for APS.

Methods: We retrospectively reviewed patients with PE and APS $(n=24)$ and those with unprovoked PE with aPL negative ( $\mathrm{n}=44)$, evaluated 2006-2016 at the Asan Medical Center. We compared patient demographics, clinical manifestations, laboratory findings, and radiological findings between the groups.

Results: On multivariate logistic regression analysis, two models of independent risk factors for APS-PE were suggested. Model I included hemoptysis (odds ratio [OR], 12.897; 95\% confidence interval [CI], 1.025-162.343), low PE severity index (OR, 0.948; 95\% CI, 0.917-0.979), and activated partial thromboplastin time (aPTT; OR, 1.166; 95\% CI, 1.040-1.307). Model II included age (OR, 0.930; 95\% CI, 0.893-0.969) and aPTT (OR, 1.104; 95\% CI, 1.000-1.217).

Conclusion: We conclude that patients with first unprovoked PE with hemoptysis and are age <40; have a low pulmonary embolism severity index, especially in risk class I-II; and/or prolonged aPTT (above 75th percentile of the reference interval), should be suspected of having APS, and undergo laboratory testing for aPL.

Keywords: Antiphospholipid Syndrome; Antibodies, Antiphospholipid; Pulmonary Embolism; Phenotype; Risk Factors

Address for correspondence: Jae Seung Lee, M.D.

Department of Pulmonary and Critical Care Medicine, Center for

Pulmonary Hypertension and Venous Thrombosis, Asan Medical Center, University of Ulsan College of Medicine, 88 Olympic-ro 43-gil, Songpagu, Seoul 05505, Korea

Phone: 82-2-3010-3994, Fax: 82-2-3010-6968

E-mail: jsdoc1186@daum.net

Received: May. 10, 2018

Revised: Jul. 22, 2018

Accepted: Oct. 16, 2018

(c) It is identical to the Creative Commons Attribution Non-Commercial License (http://creativecommons.org/licenses/by-nc/4.0/).

The Korean Academy of Tuberculosis and Respiratory Diseases.

\section{Introduction}

In patients with a first symptomatic pulmonary embolism (PE), clinicians should evaluate for predisposing factors. Unprovoked venous thromboembolism (VTE), for which there is the absence of a temporary or reversible risk factor within the 6 weeks to 3 months before diagnosis, has a moderately high risk of recurrence, which is estimated to be $10 \%$ within 1 year and $30 \%$ within 5 years ${ }^{1-3}$. The following risk factors for recurrence in patients with an unprovoked PE may help to identify those at higher long-term relative risk of recurrence: (1) one or more previous episodes of VTE, (2) antiphospholipid antibody syndrome (APS), (3) hereditary thrombophilia, or (4) residual thrombosis in the proximal veins ${ }^{3-6}$.

One of those risk factors is APS, a systemic autoimmune 
disease that is diagnosed when arterial and/or venous thrombosis, and recurrent fetal losses occurs in the presence of persistent antiphospholipid antibodies (aPL) ${ }^{7}$. APS tends to be more common in young to middle aged women. Estimates have indicated an incidence of around five new cases per 100,000 persons per year, with prevalence around $40-50$ cases per 100,000 persons ${ }^{8}$. PE is the most common pulmonary manifestation and may be the first sign of APS ${ }^{9,10}$. Patients with PE-associated APS are recommended to have extended anticoagulant therapy because of the risk of recurrent $\mathrm{VTE}^{11}$. Prothrombotic states and impaired clot dissolution are believed to contribute to the occurrence of chronic thromboembolic pulmonary hypertension in $\mathrm{APS}^{12}$.

aPL contributes to the pathogenesis of thrombosis ${ }^{13}$. Galli et al. ${ }^{14}$ reported that the odds ratios (OR) of aPL for cerebral stroke and deep vein thrombosis (DVT) ranged between 4.09 and 16.2, all with significant $95 \%$ confidence intervals (CI). Recently updated British Journal of Haematology guidelines on the investigation and management of APS recommend testing for aPL before stopping anticoagulation after unprovoked proximal DVT or PE, as a positive result favours long-term anticoagulation ${ }^{15}$. However, laboratory testing for aPL after a first symptomatic unprovoked PE remains controversial.

We hypothesized that there might be a specific clinical phenotype in patients with unprovoked acute PE indicating a need for evaluation for APS. This study investigated the clinical characteristics of PE in patients with and without APS who had unprovoked PE, looking for clinical predictors for APS.

\section{Materials and Methods}

\section{Study design and patients}

A retrospective descriptive study was conducted. All clinical, radiological, and laboratory data were retrospectively collected from medical records. We reviewed a total of 261 patients diagnosed as having had a first episode of PE between June 2006 and September 2016 at the Asan Medical Center, a 2,700-bed university-affiliated tertiary referral hospital in Seoul, Korea. PE was confirmed in all patients by pulmonary angiography or spiral computed tomography or ventilationperfusion lung scan indicating a high probability of pulmonary embolism. We tested for aPL in patients with a first unprovoked PE. If results were positive for aPL, aPL test was performed again at least 12 weeks apart. Patient records were included if the PE was confirmed to be unprovoked (i.e., idiopathic) and if laboratory testing for aPL had been performed or the patient was known to have APS. We excluded those with provoked PE in the presence of temporary or reversible risk factors (e.g., surgery, trauma, immobilization, pregnancy, oral contraceptive use, or hormone replacement therapy) within the preceding 6 weeks to 3 months ${ }^{3}$.
The study was approved by the institutional review board (IRB) of Asan Medical Center (IRB No. 2015-0516). The need for informed consent was waived owing to the retrospective nature of the study.

\section{Classification criteria for the antiphospholipid syndrome}

According to the 2006 revised APS classification criteria, APS requires the combination of at least one clinical and one laboratory criterion. Clinical criteria include vascular thrombosis (arterial, venous, or small vessel thrombosis in any tissue or organ) and/or pregnancy morbidity (one or more unexplained deaths of a morphologically normal foetus at or beyond the 10th week of gestation, or one or more premature births of a morphologically normal neonate before the 34th week of gestation because of severe pre-eclampsia/eclampsia, or recognized features of placental insufficiency, and/or three or more unexplained consecutive spontaneous abortions before the 10th week of gestation). Laboratory criteria include positive tests for circulating aPL (lupus anticoagulant [LA]; anticardiolipin antibody [ACA], IgG and/or IgM; anti- $\beta_{2}$ glycoprotein-I antibody [a $\beta 2 \mathrm{GPI}$ ], IgG and/or IgM) on two or more occasions at least 12 weeks apart?

\section{Laboratory diagnosis of APS}

LA tests were determined according to the criteria of the Subcommittee for Standardization of $\mathrm{LA}^{16}$. The diluted Russell's Viper Venom Test (dRVVT) was performed using an ACL TOP 750 automated coagulometer (Instrumentation Laboratory, Milan, Italy) with a commercial assay (HemosIL, dRVVT screen; Instrumentation Laboratory, Milan, Italy). Samples with a prolonged dRVVT that was not corrected by mixing with a normal plasma pool were tested for confirmation using an excess of phospholipids (HemosIL, dRVVT confirm; Instrumentation Laboratory, Milan, Italy). A commercial silica clotting time (SCT) with low and high concentrations of synthetic phospholipids (HemosIL, SCT screen; Instrumentation Laboratory, Milan, Italy) was used as an activator in the coagulation test. The SCT was carried out on the ACL TOP 750 automated coagulometer (Instrumentation Laboratory, Milan, Italy). Samples with a prolonged SCT that was not corrected by mixing with a normal plasma pool were tested for confirmation using a high phospholipid concentration (HemosIL, SCT confirm; Instrumentation Laboratory, Milan, Italy). ACA (IgG and IgM) and aß2GPI (IgG and IgM) were tested for using commercially assay kits (HemosIL; Instrumentation Laboratory, Bedford, MA, USA). Both tests were carried out on automated immunoassay, the HemosIL AcuStar (Instrumentation Laboratory, Bedford, MA, USA). All tests were performed according to the manufacturer's instructions. 


\section{Risk stratification}

The pulmonary embolism severity index (PESI) is a clinical prediction rule for prognosis of PE developed by Aujesky et al.$^{17}$ The PESI correlates with 30-day mortality ${ }^{3,17,18}$. The risk of early mortality in patients with acute PE was classified as high, intermediate-high, intermediate-low, and low. Risk parameters and scores included shock or hypotension, PESI class III$\mathrm{V}$ or a simplified PESI score $\geq 1$, signs of right ventricular (RV) dysfunction on an imaging test, and cardiac laboratory biomarkers. Risk-adjusted therapeutic strategies and algorithms are recommended on the basis of this risk classification ${ }^{3}$.

\section{Statistical analysis}

We compared patient demographics, clinical manifestations, laboratory findings, and radiological findings between patients with a PE associated with APS (APS-PE) and those with an unprovoked PE but who did not have APS (non-APS$\mathrm{PE}$ ). Results are expressed as means with standard deviations or median values with interquartile range (IQR) for continuous variables, and as percentages for categorical variables. For comparison of continuous variables between the APS-PE and non-APS-PE groups, parametric data were compared using independent Student's t-tests, and nonparametric data were compared using a Mann-Whitney U test. Categorical variables were compared using a chi-square or Fisher exact test. To evaluate the clinical predictors of APS-PE, univariate and multivariate logistic regression analysis was performed. Variables with a p-values of $<0.05$ as determined by univariate logistic regression analysis in consideration of multicollinearity were entered into multivariate logistic regression analysis using a backward elimination method. Assessing goodness-of-fit in logistic regression models was performed by the HosmerLemeshow goodness-of-fit test. Discrimination ability was assessed using the area under the receiver operating characteristic (ROC) curve.

All reported $p$ values were two-sided, and a p-value of $<0.05$ was considered statistically significant. Statistical analysis was performed using the IBM SPSS Statistics version 21.0 (IBM Corp., Armonk, NY, USA).

\section{Results}

\section{Patients}

Among 261 patients diagnosed with a first episode of PE, 170 were excluded from analysis because they had a temporary or reversible risk factor (Figure 1). Of 67 patients with an unprovoked PE, 23 were not tested for aPL and were excluded. So, we compared with 44 patients testing negative for aPL and 24 diagnosed with APS, either primary $(n=17)$ or in association with systemic lupus erythematous $(\mathrm{n}=7)$.

\section{Baseline characteristics}

Table 1 shows a comparison of the baseline characteristics between the APS-PE and non-APS-PE groups. Individuals in the APS-PE group were significantly younger and had more

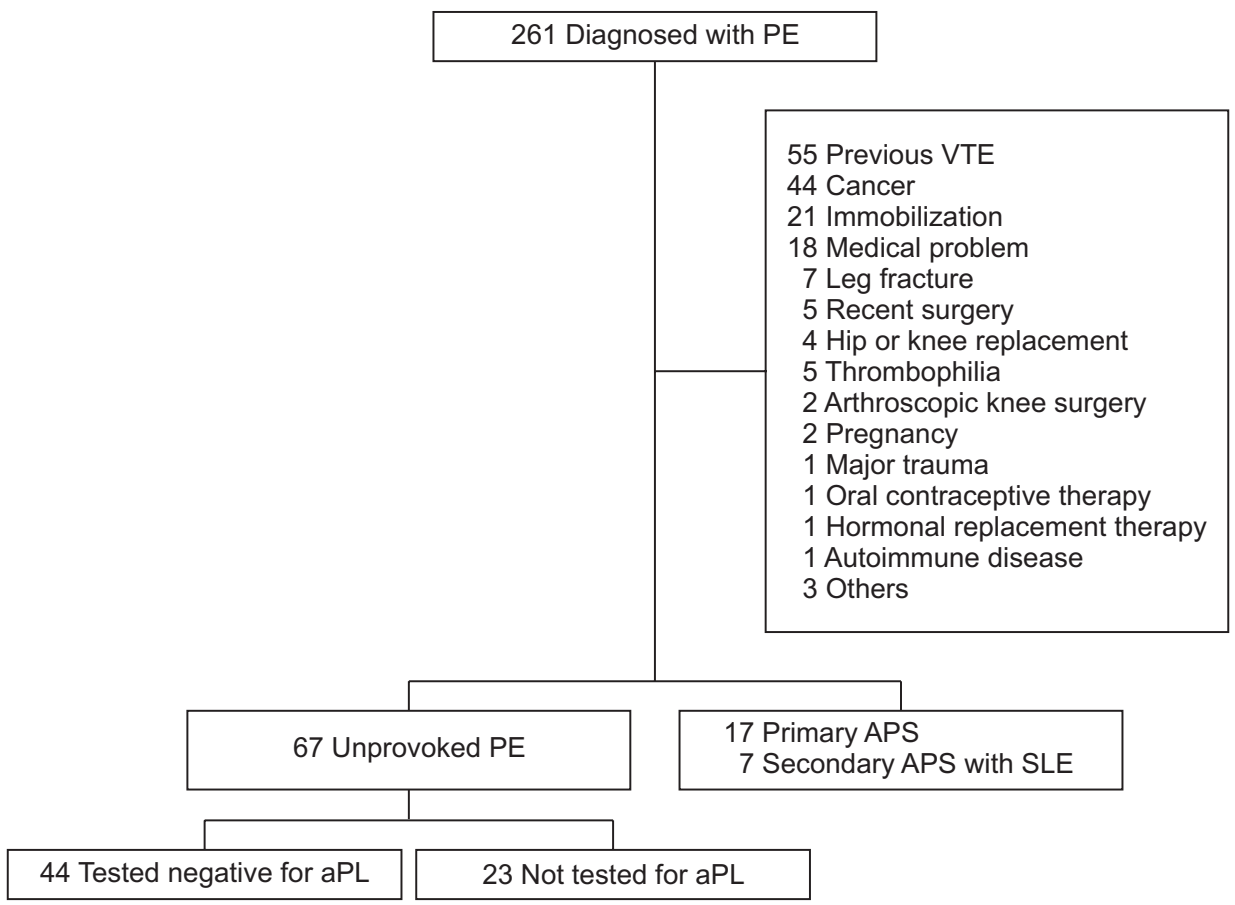

Figure 1. Flowchart of patient selection. One hundred seventy patients with predisposing factors were excluded. An additional 23 patients with unprovoked PE were excluded as they were not tested for APS. PE: pulmonary embolism; VTE: venous thromboembolism; APS: antiphospholipid antibody syndrome; SLE: systemic lupus erythematous; aPL: antiphospholipid antibody. 
Table 1. Baseline characteristics of patients with a first unprovoked PE

\begin{tabular}{|c|c|c|c|}
\hline & $\begin{array}{l}\text { APS-PE } \\
(n=24)\end{array}$ & $\begin{array}{l}\text { Non- } \\
\text { APS-PE } \\
(n=44)\end{array}$ & p-value \\
\hline Age, yr & $39.7 \pm 18.4$ & $60.4 \pm 14.0$ & $<0.001$ \\
\hline Sex & & & 0.492 \\
\hline Male & $13(54.2)$ & $20(45.5)$ & \\
\hline Female & $11(45.8)$ & $15(54.5)$ & \\
\hline Body mass index, $\mathrm{kg} / \mathrm{m}^{2}$ & $23.9 \pm 4.4$ & $25.5 \pm 3.6$ & 0.112 \\
\hline Deep vein thrombosis* & $17(70.8)$ & $25(56.8)$ & 0.256 \\
\hline History of arterial thrombosis & $3(12.5)$ & $0(0)$ & 0.017 \\
\hline $\begin{array}{l}\text { History of pregnancy } \\
\text { complications }\end{array}$ & $1(9.0)$ & $0(0)$ & 0.176 \\
\hline Presentation & & & 0.175 \\
\hline Acute signs or symptoms & $20(83.3)$ & $42(95.5)$ & \\
\hline Incidental $^{\dagger}$ & $4(16.7)$ & $2(4.5)$ & \\
\hline Diagnostic modality & & & 0.283 \\
\hline $\begin{array}{l}\text { Computed tomography } \\
\text { angiography }\end{array}$ & $22(91.7)$ & $43(97.7)$ & \\
\hline Ventilation-perfusion scan & $2(8.3)$ & $1(2.3)$ & \\
\hline
\end{tabular}

Values are presented mean \pm standard deviation or number (\%).

*Deep vein thrombosis was diagnosed by lower extremity compression ultrasonography or computed tomography venography. ${ }^{+}$Incidental PE defined as the finding of PE in situations where it was not suspected.

PE: pulmonary embolism; APS: antiphospholipid antibody syndrome.

history of arterial thrombosis.

\section{Radiological findings}

The arteries involved by the modified Boyden classification $^{19}$, involved lung lobes, and numbers of PEs by pulmonary computed tomography (CT) angiography were similar between the two groups (Table 2), but patients in the APS-PE group were more likely to have a pulmonary infarct than those in the non-APS-PE group.

\section{Clinical and laboratory criteria in APS-PE group}

As shown in Table 3, the types of aPL found in the APS-PE group included LA (in 58.3\%), ACA IgM (in 25.0\%), ACA IgG (in 29.2\%), aß2GPI IgM (in 20.8\%), and aß2GPI IgG (in 20.8\%). A history of arterial thrombosis was found in three patients (12.5\%): coronary artery disease history was found in two and cerebral infarction history in one. Among 11 female patients, one had had eclampsia. Of the 24 patients with APS, seven (29.2\%) had systemic lupus erythematosus.
Table 2. Pulmonary CT angiography findings

\begin{tabular}{|c|c|c|c|}
\hline & $\begin{array}{c}\text { APS-PE } \\
(n=22)\end{array}$ & $\begin{array}{c}\text { Non-APS-PE } \\
\quad(n=43)\end{array}$ & p-value \\
\hline Single-vessel PE & $1(4.5)$ & $6(14.0)$ & 0.408 \\
\hline Multiple-vessel PE & $21(95.5)$ & $37(86.0)$ & 0.408 \\
\hline \multicolumn{4}{|l|}{ Artery involved* } \\
\hline Pulmonary trunk & $2(9.1)$ & $2(4.7)$ & 0.599 \\
\hline $\begin{array}{l}\text { Main pulmonary } \\
\text { artery }\end{array}$ & $10(45.5)$ & $16(37.2)$ & 0.521 \\
\hline $\begin{array}{l}\text { Lobar pulmonary } \\
\text { artery }\end{array}$ & $15(68.2)$ & $34(79.1)$ & 0.335 \\
\hline $\begin{array}{l}\text { Segmental } \\
\text { pulmonary artery }\end{array}$ & $19(86.4)$ & $33(76.7)$ & 0.516 \\
\hline $\begin{array}{l}\text { Subsegmental } \\
\text { pulmonary artery }\end{array}$ & $8(36.4)$ & $12(27.9)$ & 0.485 \\
\hline \multicolumn{4}{|l|}{ Location in lung } \\
\hline Right upper lobe & $10(45.5)$ & $28(65.1)$ & 0.128 \\
\hline Right middle lobe & $9(40.9)$ & $26(60.5)$ & 0.135 \\
\hline Right lower lobe & $18(81.8)$ & $37(86.0)$ & 0.723 \\
\hline Left upper lobe & $13(59.1)$ & $27(62.8)$ & 0.772 \\
\hline Left lower lobe & $18(81.8)$ & $35(81.4)$ & 1.000 \\
\hline Pulmonary infarction $^{\dagger}$ & $11(50.0)$ & $10(23.3)$ & 0.029 \\
\hline
\end{tabular}

Values are presented as number (\%)

*Location according to the modified Boyden classification ${ }^{19}$. Defined as a wedge-shaped subpleural opacity on pulmonary CT angiography findings.

CT: computed tomography; APS: antiphospholipid antibody syndrome; PE: pulmonary embolism.

Table 3. Clinical and laboratory criteria in the APS-PE group

\begin{tabular}{|c|c|}
\hline & APS-PE $(n=24)$ \\
\hline \multicolumn{2}{|l|}{ Antiphospholipid antibodies } \\
\hline Lupus anticoagulants & $14(58.3)$ \\
\hline Anticardiolipin antibody IgM & $6(25.0)$ \\
\hline Anticardiolipin antibody IgG & $7(29.2)$ \\
\hline Anti- $\beta_{2}$ glycoprotein-I antibody IgM & $5(20.8)$ \\
\hline Anti- $\beta_{2}$ glycoprotein-I antibody IgG & $5(20.8)$ \\
\hline Arterial thrombosis* & $3(12.5)$ \\
\hline Pregnancy complications $^{\dagger}$ & $1(9.0)$ \\
\hline Secondary APS with SLE & $7(29.2)$ \\
\hline
\end{tabular}

Values are presented as number (\%).

*Two patients had coronary artery disease. One patient had cerebral infarction. ${ }^{\dagger}$ One of eleven women in the APS-PE group had eclampsia.

APS: antiphospholipid antibody syndrome; PE: pulmonary embolism; SLE: systemic lupus erythematous. 


\section{Clinical manifestations and laboratory findings}

The clinical manifestations and laboratory findings of the two groups are summarized in Table 4. patients in the APSPE group had less dyspnea but significantly more hemoptysis, unilateral leg pain, and signs of DVT than the non-APS-PE group. In addition, the APS-PE group had a significantly higher creatinine clearance, prolonged activated partial thromboplastin time (aPTT), and lower aspartate transaminase levels.

\section{Prognostic assessment}

PESI and early mortality risk are shown in Table 5 . The nonAPS-PE group had significantly higher creatine kinase-MB ( $0.65,0.30-1.60$ vs. $1.85,1.00-4.35 ; \mathrm{p}=0.003)$, PESI, and PESI severity classification than the APS-PE group.

\section{Clinical predictors for APS-PE}

On univariate logistic regression analysis, significant clinical predictors for APS were age, pulmonary infarction, dyspnea, hemoptysis, unilateral leg pain, signs of DVT, PESI, aPTT, creatinine clearance, and C-reactive protein (CRP). The risk of having APS was 11-fold higher for patients with hemoptysis (OR, 11.316; 95\% CI, 1.237-103.553) and 3-fold higher for patients with pulmonary infarction (OR, 3.400; 95\% CI, 1.1709.877), unilateral leg pain (OR, 3.400; 95\% CI, 1.170-9.877), or signs of DVT (OR, 3.545; 95\% CI, 1.236-10.170) (Table 6). The significant independent risk factors for having APS by multivariate logistic regression analysis for PESI, hemoptysis, signs of DVT, aPTT (model I) were hemoptysis (OR, 12.897; 95\% CI, 1.025-162.343), a low PESI (OR, 0.948; 95\% CI, 0.917-0.979), and aPTT (OR, 1.166; 95\% CI, 1.040-1.307) (Table 7). When model I was applied to the validation data, the area under the ROC curve was 0.868 and the p-value for the Hosmer-Leme-

Table 4. Comparison of clinical manifestations and laboratory findings between the APS-PE and non-APS-PE groups

\begin{tabular}{|c|c|c|c|}
\hline & APS-PE $(n=24)$ & Non-APS-PE $(n=44)$ & p-value \\
\hline \multicolumn{4}{|l|}{ Clinical manifestations } \\
\hline Dyspnea & $14(58.3)$ & $37(84.1)$ & 0.019 \\
\hline Pleuritic chest pain & $6(25.0)$ & $5(11.4)$ & 0.177 \\
\hline Cough & $4(16.7)$ & $2(4.5)$ & 0.175 \\
\hline Substernal chest pain & $5(20.8)$ & $9(20.5)$ & 1.000 \\
\hline Fever & $3(12.5)$ & $1(2.3)$ & 0.122 \\
\hline Hemoptysis & $5(20.8)$ & $1(2.3)$ & 0.018 \\
\hline Syncope & $2(8.3)$ & $5(11.4)$ & $>0.990$ \\
\hline Unilateral leg pain & $12(50.0)$ & $10(22.7)$ & 0.022 \\
\hline Signs of deep vein thrombosis* & $13(54.2)$ & $11(25.0)$ & 0.016 \\
\hline Hypotension $^{+}$ & $1(4.2)$ & $4(9.1)$ & 0.649 \\
\hline Heart rate, beats per minute & $90.3 \pm 14.9$ & $94.1 \pm 18.3$ & 0.396 \\
\hline \multicolumn{4}{|l|}{ Laboratory findings } \\
\hline White blood cell count, $/ \mu \mathrm{L}$ & $8,707 \pm 4,381$ & $9,792 \pm 3,535$ & 0.271 \\
\hline Platelet, $\times 10^{3} / \mu \mathrm{L}$ & $190,125 \pm 102,075$ & $205,090 \pm 62,744$ & 0.518 \\
\hline Creatinine clearance, $\mathrm{mL} / \mathrm{min} / 1.73 \mathrm{~m}^{2}$ & $114.4 \pm 25.1$ & $82.9 \pm 26.2$ & $<0.001$ \\
\hline Prothrombin time, sec & $11.8 \pm 1.2$ & $11.5 \pm 1.3$ & 0.366 \\
\hline International normalized ratio & $1.05 \pm 0.11$ & $1.03 \pm 0.11$ & 0.398 \\
\hline Activated partial thromboplastin time, sec & $31.8(27.3-35.2)$ & $27.1(25.2-28.6)$ & $<0.001$ \\
\hline Aspartate transaminase, IU/L & $22.0(19.0-29.5)$ & $28.0(21.5-45.0)$ & 0.029 \\
\hline Alanine transaminase, IU/L & $20.5(11.5-40.5)$ & $27.0(17.5-47.0)$ & 0.152 \\
\hline C-reactive protein, $\mathrm{mg} / \mathrm{dL}$ & $1.17(0.45-4.22)$ & $0.55(0.16-1.79)$ & 0.051 \\
\hline
\end{tabular}

Values are presented as number (\%), mean \pm standard deviation, or median (interquartile range).

*A palpable cord reflecting a thrombosed vein, ipsilateral edema, warmth, and/or superficial venous dilation. ${ }^{\dagger}$ Defined as systolic blood pressure $<90 \mathrm{~mm} \mathrm{Hg}$ or a systolic pressure drop by $\geq 40 \mathrm{~mm} \mathrm{Hg}$ for $>15$ minutes, if not caused by new-onset arrhythmia, hypovolemia or sepsis ${ }^{3}$. APS: antiphospholipid antibody syndrome; PE: pulmonary embolism. 
show goodness-of-fit test was 0.656. Model II included age, hemoptysis, signs of DVT, aPTT, and CRP. The independent risk factors were age (OR, 0.930; 95\% CI, 0.893-0.969) and aPTT (OR, 1.104; 95\% CI, 1.000-1.217). When model II was applied to the validation data, the area under the ROC curve was 0.819 and the p-value for the Hosmer-Lemeshow goodness-of-fit test was 0.102 .

\section{Discussion}

Our study shows which clinical factors in patients with unprovoked PE are predictive of APS, thereby suggesting which

Table 5. PESI and early mortality risk

\begin{tabular}{|lccc|}
\hline & $\begin{array}{c}\text { APS-PE } \\
(\mathbf{n = 2 4 )}\end{array}$ & $\begin{array}{c}\text { Non-APS- } \\
\text { PE (n=44) }\end{array}$ & p-value \\
\hline PESI & & & 0.039 \\
\hline Class I ( $\leq 65$ points) & $14(58.3)$ & $11(25.0)$ & \\
\hline Class II (65-85 points) & $7(29.2)$ & $13(29.5)$ & \\
\hline Class III (86-105 points) & $3(12.5)$ & $14(31.8)$ & \\
\hline Class IV (106-125 points) & $0(0)$ & $3(6.8)$ & \\
Class V (>125 points) & $0(0)$ & $3(6.8)$ & \\
\hline Average* & $58.0 \pm 21.6$ & $83.7 \pm 24.4$ & $<0.001$ \\
Early mortality risk & & & 0.072 \\
\hline High & $1(4.2)$ & $4(9.1)$ & \\
\hline Intermediate-high & $1(4.2)$ & $5(11.4)$ & \\
\hline Intermediate-low & $7(29.2)$ & $22(50.0)$ & \\
\hline Low & $15(62.5)$ & $13(29.5)$ & \\
\hline
\end{tabular}

Values are presented as number (\%) or mean \pm standard deviation. *Sum of points.

PESI: pulmonary embolism severity index; APS: antiphospholipid antibody syndrome; PE: pulmonary embolism. patients need to be evaluated for APS. The factors we found to be associated with APS were (1) younger age, (2) the presence of a pulmonary infarct on CT pulmonary angiography, (3) the absence of dyspnea, (4) the presence of hemoptysis, (5) the presence of unilateral leg pain and signs of DVT, (6) a low PESI, (7) prolonged aPTT, and (8) high creatinine clearance and CRP. Among these factors, the most sensitive predictors for APS were younger age, the presence of hemoptysis, a low PESI, and prolonged aPTT.

The prevalence of APS is approximately $40-50$ cases per 100,000 persons $\mathrm{s}^{20,21}$. APS usually occurs in young patients. Piette and Cacoub ${ }^{22}$ analyzed data on age at first thrombosis in patients with primary APS and found that the first event occurred in patients aged $<50$ years. This is in contrast to the risk for VTE in the general population which, as Anderson and

Table 6. Clinical predictors of APS on univariate logistic regression

\begin{tabular}{|lcc|}
\hline \multicolumn{1}{|c}{ Variable } & OR $(\mathbf{9 5 \%} \mathbf{C I})$ & p-value \\
\hline Age & $0.929(0.895-0.964)$ & $<0.001$ \\
Pulmonary infarction & $3.400(1.170-9.877)$ & 0.025 \\
Dyspnea & $0.265(0.084-0.832)$ & 0.023 \\
Hemoptysis & $11.316(1.237-103.553)$ & 0.032 \\
Unilateral leg pain & $3.400(1.170-9.877)$ & 0.025 \\
Signs of DVT & $3.545(1.236-10.170)$ & 0.019 \\
PESI & $0.950(0.923-0.978)$ & 0.001 \\
aPTT & $1.133(1.025-1.253)$ & 0.015 \\
Creatinine clearance & $1.057(1.027-1.088)$ & $<0.001$ \\
C-reactive protein & $1.150(1.004-1.318)$ & 0.044 \\
\hline
\end{tabular}

APS: antiphospholipid antibody syndrome; OR: odds ratio; $\mathrm{CI}$ confidence interval; DVT: deep vein thrombosis; PESI: pulmonary embolism severity index; aPTT: activated partial thromboplastin time.

Table 7. Multivariate logistic regression model of independent risk factors for PE associated with APS

\begin{tabular}{|c|c|c|c|c|}
\hline \multirow{2}{*}{ Variable } & \multicolumn{2}{|c|}{ Model I* } & \multicolumn{2}{|c|}{ Model II ${ }^{\dagger}$} \\
\hline & OR $(95 \% \mathrm{CI})$ & p-value & OR $(95 \% \mathrm{CI})$ & p-value \\
\hline Age & - & - & $0.930(0.893-0.969)$ & 0.001 \\
\hline PESI & $0.948(0.917-0.979)$ & 0.001 & - & - \\
\hline Hemoptysis & $12.897(1.025-162.343)$ & 0.048 & - & - \\
\hline Signs of DVT & - & - & - & - \\
\hline aPTT & $1.166(1.040-1.307)$ & 0.008 & $1.104(1.000-1.219)$ & 0.050 \\
\hline C-reactive protein & - & - & - & - \\
\hline
\end{tabular}

*Multivariate logistic regression analysis for PESI, hemoptysis, signs of DVT, aPTT, and C-reactive protein. ${ }^{\dagger}$ Multivariate logistic regression analysis for age, hemoptysis, signs ofDVT, aPTT, and C-reactive protein.

PE: pulmonary embolism; APS: antiphospholipid antibody syndrome; OR: odds ratio; CI: confidence interval; PESI: pulmonary embolism severity index; DVT: deep vein thrombosis; aPTT: activated partial thromboplastin time. 
Spencer $^{23}$ reported, increases in patients aged $>40$ years of age and approximately doubles with each subsequent decade. In this analysis, the mean age of the APS-PE group was 39.7 years, compared with 60.4 years in the non-APS-PE group. Therefore, if a patient aged $<40$ years presents with a first symptomatic unprovoked PE, we recommend testing for aPL.

The presenting symptoms and signs of PE are nonspecific. Our study showed that the APS-PE group had more hemoptysis and pulmonary infarction than the non-APS-PE group. The EMPEROR study reported that the most common presenting signs and symptoms of PE were dyspnea at rest (50.1\%), pleuritic chest pain (39.4\%), dyspnea on exertion (27\%), extremity edema suggestive of DVT (23.5\%), and cough without hemoptysis $(22.9 \%)$. Cough with hemoptysis was present in only $7.6 \%{ }^{24}$. He et al. ${ }^{25}$ reported that $32 \%$ patients with PE had pulmonary infarction. In our analysis, hemoptysis in the APSPE group was present in $20.8 \%$ patients, and half the APS-PE group had pulmonary infarction in pulmonary CT angiography. Pulmonary infarction occurs more commonly in patients with peripheral than central pulmonary emboli due to the dual blood supply of the lung, i.e., with occlusion of pulmonary artery branches of $\leq 3 \mathrm{~mm}$ in diameter. A wedge-shaped subpleural opacity ("Hampton hump") on X-ray has been thought by many to be diagnostic of pulmonary infarction ${ }^{25,26}$. Miniati et al. ${ }^{27}$ reported that hemoptysis in patients with pulmonary infarction was seen in 19 of 105 patients (18\%) and was more frequent than in patients without pulmonary infarction. In patients with PE who had APS in our study, pulmonary infarction was more likely to occur, which may be accompanied by hemoptysis. APS is characterized by thrombophilia with systemic hypercoagulability. Because vascular thrombosis in APS involved at any level of the vascular trees, occlusions of pulmonary arteries and bronchial arteries can occur simultaneously, which can lead to pulmonary infarction ${ }^{28}$. Therefore, hemoptysis and radiological evidence of pulmonary infarction in patients with unprovoked PE are important factors that should increase suspicion for APS.

The most common haematological manifestation in APS is thrombocytopenia, with an incidence ranging $22 \%-42 \%$ in different series. Thrombocytopenia in APS is rarely associated with haemorrhage ${ }^{29-32}$. Many patients with APS have a prolonged aPTT because LA inhibits the formation of the prothrombinase complex ${ }^{33}$. However, the presence of LA is strong risk factors for thrombosis, irrespective of the site and thrombosis type $\mathrm{e}^{14}$ and not for bleeding. In this study, the most common aPL in the APS-PE group was LA, in 14 of 24 patients (58\%). The median aPTT value in the APS-PE group was 31.8 seconds (IQR, 27.3-35.2), within the normal range. However, the median value was still significantly higher in the APS-PE than in the non-APS-PE group. On multivariate logistic regression analysis, aPTT was independently associated with APS. Thus, if patients with an unprovoked PE have an isolated prolonged aPTT above the 75th percentile of the reference inter- val, screening for aPL, especially LA, should be considered.

PESI is an accurate and reproducible tool for risk stratification in acute PE and correlates with 30-day mortality. PESI and classification of early mortality risk may help identify appropriate candidates for outpatient management or hospitalization. In recently updated guidelines, patients with a confirmed PE in PESI class I-II or low early mortality risk are recommended to have early discharge and home treatment ${ }^{3,34,35}$. Our study showed that the non-APS-PE group had a higher PESI, with a higher proportion of patients in risk classes III-V, than the APS-PE group. The distribution of early mortality risk between the APS-PE and non-APS-PE groups was not statistically significant, but $70.5 \%$ patients in the non-APS-PE group were at intermediate to high mortality risk compared with $37.5 \%$ in the APS-PE group. These patients in the non-APS-PE group were generally older than patients in the APS-PE group and had more signs of RV dysfunction. We recommend that laboratory testing for aPL should be considered for patients with unprovoked PE who have a low PESI, especially in risk class I or II.

Our study has limitations. First, it is a retrospective descriptive study from a single tertiary centre, with data collected from patients' medical records, making it susceptible to bias in data selection and analysis. Second, we did not perform a costeffectiveness analysis of testing for aPL in patients with a first unprovoked PE. Auerbach et al. ${ }^{36}$ reported that hypercoagulability testing followed by 24 months of anticoagulation was cost effective. Third, we did not analyze treatment outcomes after anticoagulation between the two groups. Fourth, the reason for the high prevalence of APS compared with recent study is unclear ${ }^{37}$.

In conclusion, this study shows that patients with a first unprovoked PE who have hemoptysis, are aged $<40$ years, had a low PESI (risk class I or II), and/or prolonged aPTT (above the 75th percentile of the reference interval) should be suspected of having APS and should undergo laboratory testing for aPL. Further research requires cost-effectiveness analysis of testing for aPL in patients with a first unprovoked PE with these factors.

\section{Authors' Contributions}

Conceptualization: Oh YM, Lee SD, Lee JS. Methodology: Lee JS. Investigation: Na YS, Lee JS. Writing - original draft preparation: Na YS. Writing - review and editing: Jang S, Hong S. Approval of final manuscript: all authors.

\section{Conflicts of Interest}

No potential conflict of interest relevant to this article was reported. 


\section{References}

1. Iorio A, Kearon C, Filippucci E, Marcucci M, Macura A, Pengo $\mathrm{V}$, et al. Risk of recurrence after a first episode of symptomatic venous thromboembolism provoked by a transient risk factor: a systematic review. Arch Intern Med 2010;170:1710-6.

2. Kearon C, Akl EA. Duration of anticoagulant therapy for deep vein thrombosis and pulmonary embolism. Blood 2014;123:1794-801.

3. Konstantinides SV, Torbicki A, Agnelli G, Danchin N, Fitzmaurice D, Galie N, et al. 2014 ESC guidelines on the diagnosis and management of acute pulmonary embolism. Eur Heart J 2014;35:3033-69, 3069a-k.

4. Zhu T, Martinez I, Emmerich J. Venous thromboembolism: risk factors for recurrence. Arterioscler Thromb Vasc Biol 2009;29:298-310.

5. Kyrle PA, Rosendaal FR, Eichinger S. Risk assessment for recurrent venous thrombosis. Lancet 2010;376:2032-9.

6. Heit JA. Predicting the risk of venous thromboembolism recurrence. Am J Hematol 2012;87 Suppl 1:S63-7.

7. Miyakis S, Lockshin MD, Atsumi T, Branch DW, Brey RL, Cervera $\mathrm{R}$, et al. International consensus statement on an update of the classification criteria for definite antiphospholipid syndrome (APS). J Thromb Haemost 2006;4:295-306.

8. Petri M. Epidemiology of the antiphospholipid antibody syndrome. J Autoimmun 2000;15:145-51.

9. Asherson RA, Cervera R. Review: antiphospholipid antibodies and the lung. J Rheumatol 1995;22:62-6.

10. Espinosa G, Cervera R, Font J, Asherson RA. The lung in the antiphospholipid syndrome. Ann Rheum Dis 2002;61:195-8.

11. Kearon C, Akl EA, Ornelas J, Blaivas A, Jimenez D, Bounameaux H, et al. Antithrombotic therapy for VTE disease: CHEST guideline and expert panel report. Chest 2016;149:315-52.

12. Mirrakhimov AE, Hill NS. Primary antiphospholipid syndrome and pulmonary hypertension. Curr Pharm Des 2014;20:545-51.

13. Ruiz-Irastorza G, Cuadrado MJ, Ruiz-Arruza I, Brey R, Crowther M, Derksen R, et al. Evidence-based recommendations for the prevention and long-term management of thrombosis in antiphospholipid antibody-positive patients: report of a task force at the 13th International Congress on antiphospholipid antibodies. Lupus 2011;20:206-18.

14. Galli M, Luciani D, Bertolini G, Barbui T. Lupus anticoagulants are stronger risk factors for thrombosis than anticardiolipin antibodies in the antiphospholipid syndrome: a systematic review of the literature. Blood 2003;101:1827-32.

15. Keeling D, Mackie I, Moore GW, Greer IA, Greaves M; British Committee for Standards in Haematology. Guidelines on the investigation and management of antiphospholipid syndrome. Br J Haematol 2012;157:47-58.

16. Pengo V, Tripodi A, Reber G, Rand JH, Ortel TL, Galli M, et al. Update of the guidelines for lupus anticoagulant detection. Subcommittee on Lupus Anticoagulant/Antiphospholipid
Antibody of the Scientific and Standardisation Committee of the International Society on Thrombosis and Haemostasis. J Thromb Haemost 2009;7:1737-40.

17. Aujesky D, Obrosky DS, Stone RA, Auble TE, Perrier A, Cornuz J, et al. Derivation and validation of a prognostic model for pulmonary embolism. Am J Respir Crit Care Med 2005;172:1041-6.

18. Chan CM, Woods C, Shorr AF. The validation and reproducibility of the pulmonary embolism severity index. J Thromb Haemost 2010;8:1509-14.

19. Boyden EA. Segmental anatomy of the lungs. New York: McGraw-Hill; 1955.

20. Mchrani T, Petri M. Epidemiology of the antiphospholipid syndrome. In: Asherson RA, editor. Handbook of systemic autoimmune diseases. Vol. 10. Amsterdam: Elsevier; 2009. p. 1334.

21. Gomez-Puerta JA, Cervera R. Diagnosis and classification of the antiphospholipid syndrome. J Autoimmun 2014;48-49:205 .

22. Piette JC, Cacoub P. Antiphospholipid syndrome in the elderly: caution. Circulation 1998;97:2195-6.

23. Anderson FA Jr, Spencer FA. Risk factors for venous thromboembolism. Circulation 2003;107(23 Suppl 1):I9-16.

24. Pollack CV, Schreiber D, Goldhaber SZ, Slattery D, Fanikos J, O'Neil BJ, et al. Clinical characteristics, management, and outcomes of patients diagnosed with acute pulmonary embolism in the emergency department: initial report of EMPEROR (Multicenter Emergency Medicine Pulmonary Embolism in the Real World Registry). J Am Coll Cardiol 2011;57:700-6.

25. He H, Stein MW, Zalta B, Haramati LB. Pulmonary infarction: spectrum of findings on multidetector helical CT. J Thorac Imaging 2006;21:1-7.

26. Tsao MS, Schraufnagel D, Wang NS. Pathogenesis of pulmonary infarction. Am J Med 1982;72:599-606.

27. Miniati M, Bottai M, Ciccotosto C, Roberto L, Monti S. Predictors of pulmonary infarction. Medicine (Baltimore) 2015;94:e1488.

28. Weng CT, Chung TJ, Liu MF, Weng MY, Lee CH, Chen JY, et al. A retrospective study of pulmonary infarction in patients with systemic lupus erythematosus from southern Taiwan. Lupus 2011;20:876-85.

29. Thrombosis and thrombocytopenia in antiphospholipid syndrome (idiopathic and secondary to SLE): first report from the Italian Registry. Italian Registry of Antiphospholipid Antibodies (IR-APA). Haematologica 1993;78:313-8.

30. Cuadrado MJ, Mujic F, Munoz E, Khamashta MA, Hughes GR. Thrombocytopenia in the antiphospholipid syndrome. Ann Rheum Dis 1997;56:194-6.

31. Cervera R, Piette JC, Font J, Khamashta MA, Shoenfeld Y, Camps MT, et al. Antiphospholipid syndrome: clinical and immunologic manifestations and patterns of disease expression in a cohort of 1,000 patients. Arthritis Rheum 2002;46:1019-27. 
32. Uthman I, Godeau B, Taher A, Khamashta M. The hematologic manifestations of the antiphospholipid syndrome. Blood Rev 2008;22:187-94.

33. Abo SM, DeBari VA. Laboratory evaluation of the antiphospholipid syndrome. Ann Clin Lab Sci 2007;37:3-14.

34. Donze J, Le Gal G, Fine MJ, Roy PM, Sanchez O, Verschuren F, et al. Prospective validation of the Pulmonary Embolism Severity Index: a clinical prognostic model for pulmonary embolism. Thromb Haemost 2008;100:943-8.

35. Streiff MB, Agnelli G, Connors JM, Crowther M, Eichinger S, Lopes R, et al. Guidance for the treatment of deep vein thrombosis and pulmonary embolism. J Thromb Thrombolysis 2016;41:32-67.

36. Auerbach AD, Sanders GD, Hambleton J. Cost-effectiveness of testing for hypercoagulability and effects on treatment strategies in patients with deep vein thrombosis. Am J Med 2004;116:816-28.

37. Kearon C, Parpia S, Spencer FA, Baglin T, Stevens SM, Bauer KA, et al. Antiphospholipid antibodies and recurrent thrombosis after a first unprovoked venous thromboembolism. Blood 2018;131:2151-60. 